\title{
Noncommutativity of the static and homogeneous limit of the axial chemical potential in the chiral magnetic effect
}

\author{
Bo Feng, ${ }^{1}$ De-fu Hou, ${ }^{2, *}$ Hai-cang Ren, ${ }^{2,3, \dagger}$ and Shuai Yuan ${ }^{1}$ \\ ${ }^{1}$ School of Physics, Huazhong University of Science and Technology, Wuhan 430074, China \\ ${ }^{2}$ Institute of Particle Physics and Key Laboratory of Quark and Lepton Physics (MOE), \\ Huazhong Normal University, Wuhan 430079, China \\ ${ }^{3}$ Physics Department, The Rockefeller University, 1230 York Avenue, New York,
} New York 10021-6399, USA

(Received 28 September 2020; accepted 10 February 2021; published 5 March 2021)

\begin{abstract}
We study the noncommutativity of different orders of zero energy-momentum limit pertaining to the axial chemical potential in the chiral magnetic effect. While this noncommutativity issue originates from the pinching singularity at one-loop order, it cannot be removed by introducing a damping term to the fermion propagators. The physical reason is that modifying the propagator alone would violate the axialvector Ward identity and as a result a modification of the longitudinal component of the axial-vector vertex is required, which contributes to chiral magnetic effect (CME). The pinching singularity with free fermion propagators was then taken over by the singularity stemming from the dressed axial-vector vertex. We show this mechanism by a concrete example. Moreover, we proved, in general, the vanishing CME in the limit order that the static limit was taken prior to the homogeneous limit in the light of Coleman-Hill theorem for a static external magnetic field. For the opposite limit that the homogeneous limit is taken first, we show that the nonvanishing CME was a consequence of the nonrenormalization of chiral anomaly for an arbitrary external magnetic field.
\end{abstract}

DOI: 10.1103/PhysRevD.103.056004

\section{INTRODUCTION}

The collective macroscopic behavior of chiral matter subject to an external magnetic field or a vorticity field, by the interplay with chiral anomaly, could manifest in anomalous transport phenomena. For instance, an electric current along the magnetic field could be induced in response to the magnetic field in the presence of a chirality imbalance, which is known as chiral magnetic effect (CME) [1-3]. It is of great interest to the phenomenology in the relativistic heavy ion collisions [4-6], as well as in the condensed matter systems, such as the Weyl and Dirac semimetals [7-10]. It is believed that the charge separation observed in the correlation of final hadrons in noncentral heavy ion collisions and the negative magnetoresistance observed in some semimetals are the consequences of the CME. Because of the noisy background, however, the

\footnotetext{
houdf@mail.ccnu.edu.cn

${ }^{\dagger}$ Renhc@mail.ccnu.edu.cn
}

Published by the American Physical Society under the terms of the Creative Commons Attribution 4.0 International license. Further distribution of this work must maintain attribution to the author(s) and the published article's title, journal citation, and DOI. Funded by SCOAP.
CME in heavy ion collisions remains controversial and the intensive investigations are ongoing [11-14].

With the chirality imbalance proxied by a constant axial chemical potential $\mu_{5}$, the chiral magnetic current in a constant magnetic field $\mathbf{B}$ takes the simple form $[2,3]$

$$
\mathbf{J}=\eta \frac{e^{2}}{2 \pi^{2}} \mu_{5} \mathbf{B},
$$

with $\eta$ a factor associated with color and flavor degrees of freedom. In the reality of heavy ion collisions, however, both magnetic field and chirality imbalance are inhomogeneous and time dependent, and (1) serves an approximation for slowly varying $\mu_{5}$ and $\mathbf{B}$. With an arbitrary magnetic field and arbitrary axial chemical potential, the chiral magnetic current in momentum representation reads

$$
J^{i}(q+k)=\eta \frac{e^{2}}{2 \pi^{2}} \mathcal{G}^{i j 0}(q, k) \mu_{5}(k) A^{j}(q),
$$

where $\mathcal{G}^{\mu \nu \rho}(q, k)$ is proportional to the Axial vector-VectorVector (AVV) three-point functions with one of the photon vertices and the axial-vector vertex bearing incoming 4-momenta $q$ and $k$. The current in the form (1) corresponds to its infrared limit, $q \rightarrow 0$ and $k \rightarrow 0$, but this limit is subtle at a nonzero temperature.

At a constant $\mu_{5}$, i.e., $k=0, \mathcal{G}^{i j 0}(q, 0)$ can be parametrized as 


$$
\mathcal{G}^{i j 0}(q, 0)=-i F(q) \epsilon^{i j k} q^{k}
$$

For the benefit of the subsequent discussions, we emphasize here that this structure solely follows from the rotation invariance and the parity oddness without applying any Ward identities [15]. It was first found in [3] that the limits $\mathbf{q} \rightarrow 0$ and $q^{0} \rightarrow 0$ do not commutate, i.e., $\lim _{\mathbf{q} \rightarrow 0} \lim _{q^{0} \rightarrow 0} F(q)=1$ and $\lim _{q^{0} \rightarrow 0} \lim _{\mathbf{q} \rightarrow 0} F(q)=1 / 3$. This noncommutativity in different order of limits was later confirmed by the calculations with Pauli-Villars (PV) [15] and lattice regularizations [16]. Note that, however, the calculations with a proper regularization give rise to different results, i.e., $\lim _{\mathbf{q} \rightarrow 0} \lim _{q^{0} \rightarrow 0} F(q)=0$ and $\lim _{q^{0} \rightarrow 0} \lim _{\mathbf{q} \rightarrow 0} F(q)=-2 / 3$. In the PV regularization scheme, for example, the extra contribution making the difference comes entirely from the regulator term.

The authors of [17] provided a resolution to this noncommutativity problem by considering the interacting chiral fermion system. Specifically, it amounts to replacing the free fermion propagator with a dressed fermion propagator incorporating a damping term in its self-energy. As a result, because of the finite relaxation time, the pinching singularity underlying the noncommutativity in different order of limits disappears. The authors therefore found that in both orders of limits the form factor $F(0)=1$. This result is also consistent with the calculation in the strong coupling regime using the AdS/CFT correspondence [18], where the limit $q \rightarrow 0$ is unambiguous. An interesting discussion of this noncommutativity problem in the framework of chiral kinetic theory including Berry curvature [19-22] was presented in [23], where a new contribution called magnetization current to CME was identified and attributed to the removal of the discontinuity of the CME conductivity in different order of limits.

Coming to the infrared limit $k=\left(k^{0}, \mathbf{k}\right) \rightarrow 0$, the explicit one-loop calculation under the PV regularization in [15] reveals the following noncommutativity. If the static limit of the chiral imbalance is prior to its homogeneity limit,

$$
\lim _{\mathbf{k} \rightarrow 0} \lim _{k^{0} \rightarrow 0} \mathcal{G}^{i j 0}(q, k)=0,
$$

for a static magnetic field in the homogeneous limit, i.e., $q=(0, \mathbf{q}) \rightarrow 0$. In the opposite order, if the homogeneity limit is prior to the static limit

$$
\lim _{k^{0} \rightarrow 0} \lim _{\mathbf{k} \rightarrow 0} \mathcal{G}^{i j 0}(q, k)=i \epsilon^{i j k} q^{k}
$$

for arbitrary $q$. The latter order of limit gives rise to the chiral magnetic current

$$
\mathbf{J}=-\eta \frac{e^{2}}{2 \pi^{2}} \mu_{5} \mathbf{B}
$$

which differs from (1) by a sign. The sign difference, however, cannot be detected with parity-even signal such as charge separation in heavy ion collisions or magnetoresistance in Weyl/Dirac semimetals. The authors of [15] related (4) to the vector Ward identity and (5) to the anomalous axial-vector Ward identity. Both identities go beyond oneloop order suggesting that the noncommutativity of the infrared limit persists to all orders.

As will be shown in the next section, the noncommutativity associated with the axial-vector vertex at one-loop level stems from the same pinching singularity as that underlying the noncommutativity of the photon vertex. A natural question that arises is why the dressed propagator fails to smear the difference between (4) and (5), and its answer together with related analysis occupy the rest of this work. Briefly speaking, a Ward identity links the longitudinal component of a vertex function with respect to the four-momentum transfer to the self-energy function of the fermion propagator attached to it. Therefore it is inconsistent to modify a fermion propagator alone. In case of the vertex of the magnetic field, only the transverse component contributes so the inconsistency does not manifest. This, however, is not the case with the axial-vector vertex. While the limit order (4) projects out the transverse component of the vertex and the inconsistency does not contribute, the opposite order of limits (5) does pick up the longitudinal component and the modification of the vertex function cannot be ignored. Through a subset of diagrams contributing to CME with the recipe [17] of the modified propagator, we shall demonstrate that the role of the pinching singularity of free propagators is taken over by the new infrared singularity of the modified axial-vector vertex and the difference between the two orders of limits (4) and (5) remains.

The rest of the paper is organized as follows: in Sec. II we shall present a one-loop calculation in order to elucidate the role of pinching singularity in the noncommutativity issue at the axial-vector vertex. A recapitulation of the Ward identity arguments in [24] in the light of the Coleman-Hill's theorem and the nonrenormalization theorem of anomaly will be presented in Sec. III for selfcontainedness. In Sec. IV, a concrete example is given for demonstrating the mechanism of the failure of the dressed fermion propagator in the noncommutativity issue. Section V concludes the paper. For the sake of simplicity, we shall mainly consider a static magnetic field. Except for Sec. III A, we shall work in the framework of the closedtime path (CTP) Green's functions which is detailed in the Appendix. Throughout the paper, we will stay with the Minkowski metric and four vectors represented by $x^{\mu}=\left(x^{0}, \mathbf{x}\right), q^{\mu}=\left(q^{0}, \mathbf{q}\right)$ with $q^{0}$ the energy. 


\section{ONE-LOOP ANALYSIS}

In this section, we shall calculate the contribution to CME from the one-loop AVV three-point function in terms of CTP Green functions at a nonzero temperature in order to exhibit the role of the pinching singularity. The CTP Green's functions [25] are generated by a path integral whose action is the integration of the classical Lagrangian along a closed time path that consists of a forward branch and a backward branch. The quantum field operator in CTP is denoted by $\phi_{a}(x)$ with $a=1,2$ labeling the forward and backward branches. For more details about CTP Green's functions, see the Appendix and Ref. [25]. The amplitude of Fig. 1 in CTP formalism reads

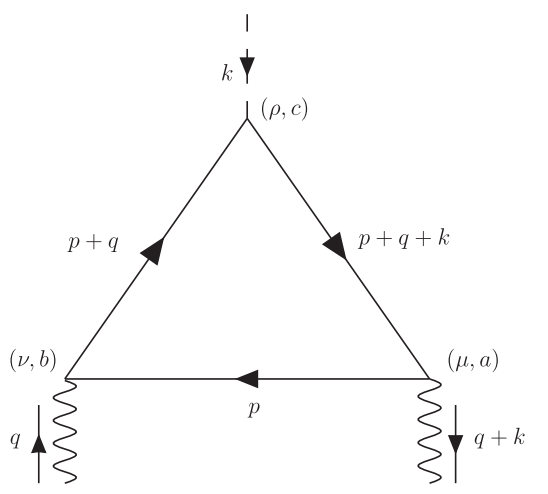

FIG. 1. The AVV triangle diagram. There is a second diagram with the photon four-momenta and polarization as well as CTP indices interchanged.

$\mathcal{G}_{a b c}^{\mu \nu \rho}(q, k)=-i e^{2} \int \frac{d^{4} p}{(2 \pi)^{4}} \operatorname{Tr}\left[\Gamma^{\mu} \eta_{a} S(p+q+k) \Gamma^{\rho 5} \eta_{c} S(p+q) \Gamma^{\nu} \eta_{b} S(p)+\Gamma^{\mu} \eta_{a} S(p+q+k) \Gamma^{\nu} \eta_{b} S(p+k) \Gamma^{\rho 5} \eta_{c} S(p)\right]$,

where the trace $\operatorname{Tr}(\ldots)$ was extended to Dirac and CTP indices. The subscripts in $\mathcal{G}_{a b c}^{\mu \nu \rho}$ are CTP indices each taking values 1 and 2 , which are projected by the $2 \times 2$ matrices

$$
\eta_{1}=\left(\begin{array}{ll}
1 & 0 \\
0 & 0
\end{array}\right), \quad \text { and } \quad \eta_{2}=\left(\begin{array}{ll}
0 & 0 \\
0 & 1
\end{array}\right)
$$

The bare vertices and propagators take the form

$$
\Gamma^{\mu}=\left(\begin{array}{cc}
\gamma^{\mu} & 0 \\
0 & -\gamma^{\mu}
\end{array}\right), \quad \Gamma^{\mu 5}=\left(\begin{array}{cc}
\gamma^{\mu} \gamma^{5} & 0 \\
0 & -\gamma^{\mu} \gamma^{5}
\end{array}\right)
$$

with the negative sign taking into account the reversed time integration along the backward branch, and

$$
S(p)=\left(\begin{array}{ll}
S_{11} & S_{12} \\
S_{21} & S_{22}
\end{array}\right)
$$

with

$$
\begin{aligned}
& S_{11}(p)=\frac{i}{\not p+i 0^{+}}-2 \pi \not p\left[\theta\left(-p_{0}\right)+\epsilon\left(p_{0}\right) f_{F}\left(p_{0}\right)\right] \delta\left(p^{2}\right), \\
& S_{12}(p)=-2 \pi \not p \epsilon\left(p_{0}\right) f_{F}\left(p_{0}\right) \delta\left(p^{2}\right), \\
& S_{21}(p)=-2 \pi \not p \epsilon\left(p_{0}\right)\left[f_{F}\left(p_{0}\right)-1\right] \delta\left(p^{2}\right), \\
& S_{22}(p)=\frac{-i}{\not p-i 0^{+}}-2 \pi \not p\left[\theta\left(-p_{0}\right)+\epsilon\left(p_{0}\right) f_{F}\left(p_{0}\right)\right] \delta\left(p^{2}\right),
\end{aligned}
$$

for massless fermions with $f_{F}(x)=1 /\left(e^{\beta x}+1\right)$, the Fermi-Dirac distribution function at temperature $T=1 / \beta$. We also defined $\theta(x)=1$ if $x>0$ and vanishing otherwise as well as $\epsilon(x)= \pm 1$ for positive and negative $x$, respectively. The AVV three-point function underlying retarded responses of the CME current to the magnetic field and axial chemical potential was obtained by restricting the electric current operator within the forward branch and summing up the rest CTP indices, i.e., 


$$
\begin{aligned}
\mathcal{G}_{R}^{i j 0}(q, k)= & \sum_{b, c} \mathcal{G}_{1 b c}^{i j 0}(q, k) \\
= & -i e^{2} \int \frac{d^{4} p}{(2 \pi)^{4}} \operatorname{Tr} \gamma^{i}\left[S_{R}(p+q+k) \gamma^{0} \gamma^{5} S_{R}(p+q) \gamma^{j} S_{C}(p)+S_{C}(p+q+k) \gamma^{0} \gamma^{5} S_{A}(p+q) \gamma^{j} S_{A}(p)\right. \\
& +S_{R}(p+q+k) \gamma^{0} \gamma^{5} S_{C}(p+q) \gamma^{j} S_{A}(p)+S_{C}(p+q+k) \gamma^{j} S_{A}(p+k) \gamma^{0} \gamma^{5} S_{A}(p) \\
& \left.+S_{R}(p+q+k) \gamma^{j} S_{C}(p+k) \gamma^{0} \gamma^{5} S_{A}(p)+S_{R}(p+q+k) \gamma^{j} S_{R}(p+k) \gamma^{0} \gamma^{5} S_{C}(p)\right],
\end{aligned}
$$

where we have switched to the physical representation of CTP formalism and the trace tr(...) extends to Dirac indices only. The physical representation can be obtained from (10) by an orthogonal transformation given in the Appendix and we have the retarded and advanced fermion propagators, and the correlator given by

$$
\begin{gathered}
S_{R}(p)=\frac{i}{\not p+i 0^{+} \gamma^{0}}, \\
S_{A}(p)=\frac{i}{\not p-i 0^{-} \gamma^{0}}, \\
S_{C}(p)=2 \pi \not p\left(1-2 f_{F}\left(p^{0}\right)\right) \delta\left(p^{2}\right) .
\end{gathered}
$$

Substituting the Kubo-Martin-Schwinger (KMS) relation $S_{C}(p)=\left[1-2 f_{F}\left(p^{0}\right)\right]\left[S_{R}(p)-S_{A}(p)\right]$, we have

$$
\begin{aligned}
\mathcal{G}_{R}^{i j 0}(q, k)= & -i e^{2} \int \frac{d^{4} p}{(2 \pi)^{4}} \operatorname{Tr} \gamma^{i} \\
& \times\left\{f _ { F } ( p ^ { 0 } ) \left[S_{R}(p+q+k) \gamma^{0} \gamma^{5} S_{R}(p+q) \gamma^{j} S_{R}(p)+S_{R}(p+q+k) \gamma^{j} S_{R}(p+k) \gamma^{0} \gamma^{5} S_{R}(p)\right.\right. \\
& \left.-S_{R}(p+q+k) \gamma^{0} \gamma^{5} S_{R}(p+q) \gamma^{j} S_{A}(p)-S_{R}(p+q+k) \gamma^{j} S_{R}(p+k) \gamma^{0} \gamma^{5} S_{A}(p)\right] \\
& -f_{F}\left(p^{0}+q^{0}+k^{0}\right)\left[S_{A}(p+q+k) \gamma^{0} \gamma^{5} S_{A}(p+q) \gamma^{j} S_{A}(p)+S_{A}(p+q+k) \gamma^{j} S_{A}(p+k) \gamma^{0} \gamma^{5} S_{A}(p)\right. \\
& \left.-S_{R}(p+q+k) \gamma^{0} \gamma^{5} S_{A}(p+q) \gamma^{j} S_{A}(p)-S_{R}(p+q+k) \gamma^{j} S_{A}(p+k) \gamma^{0} \gamma^{5} S_{A}(p)\right] \\
& +f_{F}\left(p^{0}+q^{0}\right)\left[S_{R}(p+q+k) \gamma^{0} \gamma^{5} S_{R}(p+q) \gamma^{j} S_{A}(p)-S_{R}(p+q+k) \gamma^{0} \gamma^{5} S_{A}(p+q) \gamma^{j} S_{A}(p)\right] \\
& \left.-f_{F}\left(p^{0}+k^{0}\right)\left[S_{R}(p+q+k) \gamma^{j} S_{A}(p+k) \gamma^{0} \gamma^{5} S_{A}(p)-S_{R}(p+q+k) \gamma^{j} S_{R}(p+k) \gamma^{0} \gamma^{5} S_{A}(p)\right]\right\} .
\end{aligned}
$$

For a static magnetic field, i.e., $q=(0, \mathbf{q})$, the above equation is simplified to

$$
\begin{aligned}
\mathcal{G}_{R}^{i j 0}(q, k)= & -i e^{2} \int \frac{d^{4} p}{(2 \pi)^{4}} \operatorname{Tr} \gamma^{i} \\
& \times\left\{f_{F}\left(p^{0}\right)\left[S_{R}(p+q+k) \gamma^{0} \gamma^{5} S_{R}(p+q) \gamma^{j} S_{R}(p)+S_{R}(p+q+k) \gamma^{j} S_{R}(p+k) \gamma^{0} \gamma^{5} S_{R}(p)\right]\right. \\
& +\left[f_{F}\left(p^{0}+k^{0}\right)-f_{F}\left(p_{0}\right)\right]\left[S_{R}(p+q+k) \gamma^{0} \gamma^{5} S_{A}(p+q) \gamma^{j} S_{A}(p)+S_{R}(p+q+k) \gamma^{j} S_{R}(p+k) \gamma^{0} \gamma^{5} S_{A}(p)\right] \\
& \left.-f_{F}\left(p^{0}+k^{0}\right)\left[S_{A}(p+q+k) \gamma^{0} \gamma^{5} S_{A}(p+q) \gamma^{j} S_{A}(p)+S_{A}(p+q+k) \gamma^{j} S_{A}(p+k) \gamma^{0} \gamma^{5} S_{A}(p)\right]\right\}
\end{aligned}
$$

The noncommutativity in the orders of limits $k \rightarrow 0$ stems from the pinching singularity of the terms of the structure $S_{R} \gamma^{0} \gamma^{5} S_{A}$. If $k^{0} \rightarrow 0$ at a nonzero $\mathbf{k}$, the poles of $S_{R}$ and $S_{A}$ on the complex $p^{0}$ plane are separate and prefactor $\left[f_{F}\left(p^{0}+\right.\right.$ $\left.\left.k^{0}\right)-f_{F}\left(p^{0}\right)\right]$ vanishes. The vanishing prefactor in this case renders the pinching not contributing when the limit $\mathbf{k} \rightarrow 0$ was taken afterwards and we end up with

$$
\lim _{\mathbf{k} \rightarrow 0} \lim _{k^{0} \rightarrow 0} \mathcal{G}_{R}^{i j 0}(q, k)=e^{2} \int \frac{d^{4} p}{(2 \pi)^{4}} f_{F}\left(p^{0}\right) \frac{\partial}{\partial p^{0}} \operatorname{Tr} \gamma^{i}\left[S_{R}(p+q) \gamma^{5} \gamma^{j} S_{R}(p)-S_{A}(p+q) \gamma^{5} \gamma^{j} S_{A}(p)\right]
$$

where the identities 


$$
\begin{aligned}
\frac{\partial}{\partial p^{0}} S_{R(A)}(p) & =i S_{R(A)}(p) \gamma^{0} S_{R(A)}(p), \\
\gamma^{5} S_{R(A)}(p) & =-S_{R(A)}(p) \gamma^{5}
\end{aligned}
$$

are employed. With a PV regularization, an expression with $S_{R}$ and $S_{A}$ in (17) replaced by massive propagators

$$
\begin{gathered}
S_{R}\left(p \mid M_{s}\right)=\frac{i}{\not p+i 0^{+} \gamma^{0}-M_{s}}, \\
S_{A}\left(p \mid M_{s}\right)=\frac{i}{\not p-i 0^{-} \gamma^{0}-M_{s}}
\end{gathered}
$$

has to be subtracted from (17). As the second identity in (19) becomes

$$
\gamma^{5} S_{R(A)}\left(p \mid M_{s}\right)=-S_{R(A)}\left(p \mid M_{s}\right) \gamma^{5}+\frac{2 i M_{s} \gamma^{5}}{\left(p^{0} \pm i 0^{+}\right)^{2}-\mathbf{p}^{2}-M_{s}^{2}},
$$

for massive propagators, we find the regularized version of (18),

$$
\begin{aligned}
\lim _{\mathbf{k} \rightarrow 0} \lim _{k^{0} \rightarrow 0} \mathcal{G}_{R}^{i j 0}(q, k)= & e^{2} \int \frac{d^{4} p}{(2 \pi)^{4}} f_{F}\left(p^{0}\right) \frac{\partial}{\partial p^{0}}\left\{\operatorname{Tr} \gamma^{i}\left[S_{R}(p+q) \gamma^{5} \gamma^{j} S_{R}(p)-S_{A}(p+q) \gamma^{5} \gamma^{j} S_{A}(p)\right]\right. \\
& \left.-\sum_{s} C_{s} \operatorname{Tr} \gamma^{i}\left[S_{R}\left(p+q \mid M_{s}\right) \gamma^{5} \gamma^{j} S_{R}\left(p \mid M_{s}\right)-S_{A}\left(p+q \mid M_{s}\right) \gamma^{5} \gamma^{j} S_{A}\left(p \mid M_{s}\right)\right]\right\} \\
& -2 i \sum_{s} C_{s} M_{s} \int \frac{d^{4} p}{(2 \pi)^{4}} f_{F}\left(p^{0}\right)\left\{\left[\Delta_{R}\left(p+q \mid M_{s}\right)+\Delta_{R}\left(p \mid M_{s}\right)\right] \operatorname{Tr} \gamma^{i}\left[S_{R}(p+q) \gamma^{0} \gamma^{5} \gamma^{j} S_{R}(p)\right]\right. \\
& \left.-\left[\Delta_{A}\left(p+q \mid M_{s}\right)+\Delta_{A}\left(p \mid M_{s}\right)\right] \operatorname{Tr} \gamma^{i}\left[S_{A}(p+q) \gamma^{0} \gamma^{5} \gamma^{j} S_{A}(p)\right]\right\}
\end{aligned}
$$

with

$$
\Delta_{(R, A)}(p \mid M)=\frac{i}{\left(p^{0} \pm i 0^{+}\right)^{2}-\mathbf{p}^{2}-M_{s}^{2}},
$$

and $\sum_{s} C_{s}=1$. We choose $C_{s}$ such that the $p^{0}$-integration by part of the first two lines legitimate, which transfers $\frac{\partial}{\partial p^{0}}$ to $f_{F}\left(p^{0}\right)$. Then the $p^{0}$ integral takes the form

$$
\int_{-\infty}^{\infty} d p^{0}\left[F\left(p^{0}+i 0^{+}\right)-F\left(p^{0}-i 0^{+}\right)\right]=\oint_{C} d p^{0} F\left(p^{0}\right),
$$

with $C$ a contour going around the entire real axis of the $p^{0}$ plane clockwise. Consequently, all we need are the residues of poles of $F\left(p^{0}\right)$ along the real axis. In the limit of infinite regulator masses, only the massless poles contribute to the integral with the integrand $\propto \frac{d f_{F}}{d p^{0}}$ and only the regulator poles with $p^{0}<0\left(f_{F}\left(p^{0}\right) \rightarrow 1\right)$ to the integral in the last line of (23). The two contributions cancel each other when $q=(0, \mathbf{q}) \rightarrow 0$, leaving a null chiral magnetic current. [The illustration of this cancellation can be seen from the example given in Sec. III A from (38) to (48)].

In the opposite order of limit, starting with $\mathbf{k}=0$, we use the relation [26]

$$
\begin{aligned}
S_{R}(p+k) \gamma^{0} \gamma^{5} S_{A}(p)= & S_{R}(p+k) \gamma^{0} \gamma^{5} S_{R}(p) \\
& +\frac{i}{k^{0}}\left[S_{R}(p)-S_{A}(p)\right] \gamma^{5} .
\end{aligned}
$$

Then in the limit $k^{0} \rightarrow 0$, the poles of $S_{R}(p+k)$ and $S_{R}(p)$ of the first term coalesce below the path of $p^{0}$ integration. As one is free to deform the integration path away from the double poles, this term does not contribute when multiplied by the vanishing prefactor $\left[f_{F}\left(p^{0}+k^{0}\right)-f_{F}\left(p^{0}\right)\right]$. On the other hand, the $1 / k^{0}$ of the second term together with the prefactor generates a nonzero contribution $-i\left(\partial f_{F}\left(p^{0}\right) / \partial p^{0}\right)\left[S_{R}(p)-S_{A}(p)\right]$ in the limit $k^{0} \rightarrow 0$ and we obtain that:

$$
\lim _{k^{0} \rightarrow 0} \lim _{\mathbf{k} \rightarrow 0} \mathcal{G}_{R}^{i j 0}(q, k)=e^{2} \int \frac{d^{4} p}{(2 \pi)^{4}} \frac{\partial}{\partial p^{0}}\left\{f_{F}\left(p^{0}\right) \operatorname{Tr} \gamma^{i}\left[S_{R}(p+q) \gamma^{5} \gamma^{j} S_{R}(p)-S_{A}(p+q) \gamma^{5} \gamma^{j} S_{A}(p)\right]\right\}
$$


When (17) is PV-regularized, the regulators do not contribute the integral with $\left[f_{F}\left(p^{0}+k^{0}\right)-f_{F}\left(p^{0}\right)\right]$ in the integrand as $M_{s} \rightarrow \infty$. This follows from the observation that the pinching singularity occurs at the poles of the propagators. In the infinite mass limit of the regulators, their poles move to infinity, where $\left[f_{F}\left(p^{0}+k^{0}\right)-\right.$ $\left.f_{F}\left(p^{0}\right)\right] \rightarrow 0$. Consequently, the regulator contribution is independent of the order of limits. However, because of (27), the massless contribution associated with $\frac{d f_{F}}{d p^{0}}$ is absent, and a nonzero chiral magnetic current emerges from the regulator contribution alone.

It appears that the pinching singularity can be removed by introducing damping term in the fermion propagator $[17,27,28]$, i.e.,

$$
\begin{aligned}
& S_{R}(p) \rightarrow \mathcal{S}_{R}(p)=\frac{i}{\not p+\frac{i}{\tau} \gamma^{0}}, \\
& S_{A}(p) \rightarrow \mathcal{S}_{A}(p)=\frac{i}{\not p-\frac{i}{\tau} \gamma^{0}} .
\end{aligned}
$$

Then the poles of $\mathcal{S}_{R}(p+k)$ and $\mathcal{S}_{A}(p)$ will never coalesce in any orders of limit $k \rightarrow 0$ and the noncommutativity disappears. While this approach of smearing the noncommutativity of limits works for the magnetic vertex, it violates the axial-vector Ward identity for the $A_{\mu} \bar{\psi} \gamma^{\mu} \psi$ vertex (with $A^{\mu}$ and $\bar{\psi}, \psi$ axial-vector and fermion fields, respectively) in our case as will be demonstrated in subsequent sections.

The conclusion of our one-loop analysis is not tied to the PV-regularization scheme employed above. The proof of the Coleman-Hill-like theorem that underlies (4) does not specify a particular regularization and (5) is a direct consequence of the axial anomaly. Therefore the noncommutativity of different orders of limit of the form (4) and (5) is valid for any UV regularization that maintains the electromagnetic gauge invariance [29]. Nevertheless, the PV regularization appears to be the simplest approach for the explicit demonstration in terms of Feynman diagrams.

\section{A GENERAL ANALYSIS}

In this section, we shall prove that the noncommutativity issue at the axial-vector vertex persists to all orders of perturbations. In one order of limits where the static limit is taken prior to the spatial homogeneity limit, we shall follow the argument of Coleman-Hill theorem [30]. In the opposite order of limits where the spatial homogeneity limit is taken first, we shall extend the diagrammatical technique employed to derive the anomalous Ward identity at zero temperature [31] to CTP Green's functions. It is convenient to revert to the conventional notation pertaining to the three-point function underlying the axial anomaly, i.e., $\Lambda^{\mu \nu \rho}\left(q_{1}, q_{2}\right)$ with $q_{1}$ and $q_{2}$ the four-momenta of two outgoing photons. In terms of the external momentum setup in Fig. 1, $\mathcal{G}^{\mu \nu \rho}(q, k)=\Lambda^{\mu \nu \rho}(q+k,-q)$ with $k=q_{1}+q_{2}$ and $q=-q_{2}$. In the rest of this section $\Lambda^{\mu \nu \rho}(q+k,-q)$ will include all higher order corrections of Fig. 1.

\section{A. The order of $\operatorname{limit}_{\lim _{k \rightarrow 0}} \lim _{k^{0} \rightarrow 0}$}

Assuming a static magnetic field, i.e., $q^{0}=0$, the first limit $k^{0} \rightarrow 0$ renders all external momenta static and the system is in thermal equilibrium. The general three-point function $\Lambda^{i j 0}(q+k,-q)$ could be evaluated by means of the Matsubara formalism. Let

$$
\Gamma^{i j}\left(\mathbf{q}_{1}, \mathbf{q}_{2}\right) \equiv \lim _{k^{0} \rightarrow 0} \Lambda^{i j 0}(q+k,-q)
$$

We have, in the limit $\mathbf{k} \rightarrow 0$,

Theorem. $-\Gamma^{i j}(q, q)=\mathcal{O}\left(|\mathbf{q}|^{2}\right)$ as $\mathbf{q} \rightarrow 0$.

Proof.-Consider the set of AVV diagrams for $\Gamma^{i j}\left(\mathbf{q}_{1}, \mathbf{q}_{2}\right)$ where the outgoing photon with 4-momentum $\left(0, \mathbf{q}_{2}\right)$ is attached to a particular fermion loop $\mathcal{L}$ of $n$ vertices (photons or the axial-vector corresponding to $\mu_{5}$ ). There are $n$ internal fermion lines of $\mathcal{L}$ to which the outgoing photon can be attached to, resulting in $n$ such diagrams for $\Gamma^{i j}\left(\mathbf{q}_{1}, \mathbf{q}_{2}\right)$. Denote by $G^{i}\left(\mathbf{q}_{1}\right)$ the amplitude of the diagram prior to attachment (the progenitor $\Gamma^{i j}$ according to [30]) and write

$$
G^{i}\left(\mathbf{q}_{1}\right)=\int \frac{d^{3} \mathbf{p}}{(2 \pi)^{3}} F^{i}\left(\mathbf{p}, \mathbf{q}_{1}\right),
$$

where $\mathbf{p}$ is the momentum running through the fermion loop $\mathcal{L}$ and all other loop momentum integration as well as all Matsubara summations are included in $F^{i}\left(\mathbf{p}, \mathbf{q}_{1}\right)$. The axial vertex, i.e., $\mu_{5}$ vertex, is not shown explicitly in $G^{i}\left(\mathbf{q}_{1}\right)$. In the presence of the gauge invariant regulator, e.g., Pauli-Villars regulator, shifting integration momentum is legitimate, we have

$$
G^{i}\left(\mathbf{q}_{1}\right)=\int \frac{d^{3} \mathbf{p}}{(2 \pi)^{3}} F^{i}\left(\mathbf{p}+\boldsymbol{\delta}, \mathbf{q}_{1}\right),
$$

with $\boldsymbol{\delta}$ an arbitrary constant vector. Consequently, all terms of the Taylor expansion of (32) in nonzero powers of $\boldsymbol{\delta}$ vanish. To the linear power

$$
\delta_{j} \int \frac{d^{3} \mathbf{p}}{(2 \pi)^{3}} \frac{\partial}{\partial p_{j}} F^{i}\left(\mathbf{p}, \mathbf{q}_{1}\right)=0 .
$$

Since $\delta$ is nonzero and its direction is arbitrary, one has

$$
\int \frac{d^{3} \mathbf{p}}{(2 \pi)^{3}} \frac{\partial}{\partial p_{j}} F^{i}\left(\mathbf{p}, \mathbf{q}_{1}\right)=\Gamma^{i j}\left(\mathbf{q}_{1}, 0\right)=0 .
$$

The first equality follows from the observation that taking derivative with respect to the loop momentum amounts to attach a photon vertex of zero outgoing momentum to each internal fermion line in succession because of 


$$
\frac{\partial}{\partial p_{j}} S(p)=S(p) \gamma^{j} S(p)
$$

Repeating the same argument for the photon vertex of incoming 4-momentum $\left(0, \mathbf{q}_{1}\right)$, we end up with

$$
\Gamma^{i j}\left(0, \mathbf{q}_{2}\right)=0 .
$$

Consequently $\Gamma^{i j}(0,0)=0$ and

$\left.\frac{\partial \Gamma_{i j}}{\partial q_{k}}\right|_{\mathbf{q}=0}=\left.\frac{\partial}{\partial q_{1 k}} \Gamma_{i j}\left(\mathbf{q}_{1}, 0\right)\right|_{\mathbf{q}_{1}=0}+\left.\frac{\partial}{\partial q_{2 k}} \Gamma_{i j}\left(0, \mathbf{q}_{2}\right)\right|_{\mathbf{q}_{2}=0}=0$
The theorem is thereby proved. The validity of this theorem requires at least two independent external spatial momenta which is the case for a three-point function. Also, the presence of axial-vector vertices is not essential other than providing an independent momentum for its proof, unlike the anomaly nonrenormalization theorem presented in Sec. III B. As a corollary of the theorem, the chiral magnetic current under a constant magnetic field vanishes with this order of limits.

As an illustration of the afore proved theorem, we shall calculate explicitly the contribution of the one-loop triangle diagram to the chiral magnetic current shown in Fig. 1 with four-momenta $q=(0, \mathbf{q})$ and $k=(0, \mathbf{k})$ :

$$
\begin{aligned}
\Lambda^{i j 0}(q+k,-q)= & i e^{2} \mu_{5} \frac{1}{\beta} \sum_{n} \int \frac{d^{3} \mathbf{p}}{(2 \pi)^{3}}\left\{\operatorname{tr}\left[\gamma^{i} S(p+q+k \mid 0) \gamma^{0} \gamma^{5} S(p+q \mid 0) \gamma^{j} S(p \mid 0)\right]\right. \\
& \left.-\sum_{s} C_{s} \operatorname{tr}\left[\gamma^{i} S\left(p+q+k \mid M_{s}\right) \gamma^{0} \gamma^{5} S\left(p+q \mid M_{s}\right) \gamma^{j} S\left(p \mid M_{s}\right)\right]+((q+k) \leftrightarrow-q, i \leftrightarrow j)\right\},
\end{aligned}
$$

with

$$
S(p \mid m)=\frac{i}{\not p-m}
$$

the free propagator for quarks. Note that we have regularized the amplitude by PV regularization with $\sum_{s} C_{s}=1$ and $M_{s} \rightarrow \infty$ after the integration. In the limit $\mathbf{k} \rightarrow 0$, we have

$$
\begin{aligned}
\Lambda^{i j 0}(q,-q)= & i e^{2} \mu_{5} \frac{1}{\beta} \sum_{n} \int \frac{d^{3} \mathbf{p}}{(2 \pi)^{3}}\left\{\operatorname{tr}\left[\gamma^{i} S(p+q \mid 0) \gamma^{0} \gamma^{5} S(p+q \mid 0) \gamma^{j} S(p \mid 0)\right]\right. \\
& \left.-\sum_{s} C_{s} \operatorname{tr}\left[\gamma^{i} S\left(p+q \mid M_{s}\right) \gamma^{0} \gamma^{5} S\left(p+q \mid M_{s}\right) \gamma^{j} S\left(p \mid M_{s}\right)\right]+(q \leftrightarrow-q, i \leftrightarrow j)\right\} .
\end{aligned}
$$

Using the identity

$$
S(p \mid m)\left(i \gamma^{0} \gamma^{5}\right) S(p \mid m)=-\frac{2 m}{p^{2}-m^{2}} S(p \mid m) \gamma^{0} \gamma^{5}-\frac{\partial S(p \mid m)}{\partial p^{0}} \gamma^{5}
$$

we find that

$$
\begin{aligned}
\Lambda^{i j 0}(q,-q) & =-e^{2} \mu_{5} \frac{1}{\beta} \sum_{n} \int \frac{d^{3} \mathbf{p}}{(2 \pi)^{3}}\left\{\operatorname{tr}\left[\gamma^{i} \frac{\partial S(p+q \mid 0)}{\partial p^{0}} \gamma^{5} \gamma^{j} S(p \mid 0)\right]\right. \\
& \left.-\sum_{s} C_{s} \operatorname{tr}\left[\gamma^{i}\left(\frac{2 M_{s}}{(p+q)^{2}-M_{s}^{2}} S\left(p+q \mid M_{s}\right) \gamma^{0} \gamma^{5}+\frac{\partial S\left(p+q \mid M_{s}\right)}{\partial p^{0}} \gamma^{5}\right) \gamma^{j} S\left(p \mid M_{S}\right)\right]+(q \leftrightarrow-q, i \leftrightarrow j)\right\},
\end{aligned}
$$

where the derivative with respect to $p^{0}$ is evaluated at the Matsubara frequency $p^{0}=i(2 n+1) \pi T$. Since the integral is properly regularized, one can shift the momentum in the last terms in parentheses by $p \rightarrow p+q$ and has 


$$
\begin{aligned}
\Lambda^{i j 0}(q,-q)= & -e^{2} \mu_{5} \frac{1}{\beta} \sum_{n} \int \frac{d^{3} \mathbf{p}}{(2 \pi)^{3}}\left\{\frac{\partial}{\partial p^{0}} \Xi^{i j}(p, p+q \mid 0)-\sum_{s} C_{s} \frac{\partial}{\partial p^{0}} \Xi^{i j}\left(p, p+q \mid M_{s}\right)\right. \\
& \left.-\sum_{s} C_{s}\left[\frac{2 M_{s}}{p^{2}-M_{s}^{2}} \Theta^{i j}\left(p, p+q \mid M_{s}\right)+\frac{2 M_{s}}{(p+q)^{2}-M_{s}^{2}} \Theta^{j i}\left(p+q, p \mid M_{s}\right)\right]\right\},
\end{aligned}
$$

with

$$
\Xi^{i j}(p, p+q \mid m)=\operatorname{tr}\left[\gamma^{i} S(p \mid m) \gamma^{5} \gamma^{j} S(p+q \mid m)\right],
$$

and

$$
\Theta^{i j}(p, p+q \mid m)=\operatorname{tr}\left[\gamma^{i} S(p \mid m) \gamma^{0} \gamma^{5} \gamma^{j} S(p+q \mid m)\right] .
$$

Although the first term on the RHS of (43) is a total derivative, its contribution does not vanish at nonzero temperature due to the distribution function that emerges from converting the Matsubara summation to a contour integral. We have

$$
\begin{aligned}
\left.\frac{1}{\beta} \sum_{n} \frac{\partial}{\partial p^{0}} \Xi^{i j}(p, p+q \mid 0)\right|_{p^{0}=i(2 n+1) \pi T} & =-\frac{1}{2 \pi i} \oint_{C} d p^{0} \frac{\partial}{\partial p^{0}} \Xi^{i j}(p, p+q \mid 0) f_{F}\left(p^{0}\right) \\
& =\frac{1}{2 \pi i} \oint_{C} d p^{0} \Xi^{i j}(p, p+q \mid 0) \frac{\partial f_{F}\left(p^{0}\right)}{\partial p^{0}} \\
& =2 q_{k} \epsilon^{i j k} \frac{\beta}{\pi} \oint_{C} d p^{0} \frac{p^{0}}{\left[\left(p^{0}\right)^{2}-\mathbf{p}^{2}\right]^{2}} \frac{e^{\beta p^{0}}}{\left(1+e^{\beta p^{0}}\right)^{2}}+\mathcal{O}\left(\mathbf{q}^{2}\right)
\end{aligned}
$$

In the last step, we have taken the limit $\mathbf{q} \rightarrow 0$ and kept only the linear term in $\mathbf{q}$. Carrying out the integrals, we obtain

$$
-\left.e^{2} \mu^{5} \frac{1}{\beta} \sum_{n} \int \frac{d^{3} \mathbf{p}}{(2 \pi)^{3}} \frac{\partial}{\partial p^{0}} \Xi^{i j}(p, p+q \mid 0)\right|_{p^{0}=i(2 n+1) \pi T}=-i \frac{e^{2}}{2 \pi^{2}} \mu^{5} q_{k} \epsilon^{i j k} \text {. }
$$

The contribution of the PV regulators on the RHS of (43) can be calculated without employing the Matsubara formalism even at nonzero temperature because of the large regulator mass $M_{s}$ in the quark propagators. Then the total derivative vanishes and we end up with

$$
\begin{aligned}
& e^{2} \mu^{5} \sum_{s} C_{s} \int \frac{d^{4} p}{(2 \pi)^{4}}\left[\frac{2 M_{s}}{p^{2}-M_{s}^{2}} \Theta^{i j}\left(p, p+q \mid M_{s}\right)+\frac{2 M_{s}}{(p+q)^{2}-M_{s}^{2}} \Theta^{j i}\left(p+q, p \mid M_{s}\right)\right] \\
& \quad=-16 i e^{2} \mu_{5} q_{k} \epsilon^{i j k} \sum_{s} C_{s} M_{s}^{2} \int \frac{d^{4} p}{(2 \pi)^{4}} \frac{1}{\left(p^{2}-M_{s}^{2}\right)^{2}\left[(p+q)^{2}-M_{s}^{2}\right]}+\mathcal{O}\left(\mathbf{q}^{2}\right) \\
& \quad=i \frac{e^{2}}{2 \pi^{2}} \mu_{5} q_{k} \epsilon^{i j k}
\end{aligned}
$$

where we used the fact that $\sum_{s} C_{s}=1$. The contribution of the PV regulators (48) cancels that of the unregularized part (47) in the one-loop triangle diagram. Therefore, for a properly regularized AVV three-point function, the CME current vanishes in the order of limits $\lim _{\mathbf{k} \rightarrow 0} \lim _{k^{0} \rightarrow 0}$ for a static external magnetic field up to the linear order in its spatial momentum q. This is exactly the expected result from the theorem above.

\section{B. The order of $\operatorname{limit}_{\lim _{k^{0} \rightarrow 0}} \lim _{k \rightarrow 0}$}

We shall consider in this section the other order of limit, i.e., taking the spatial homogeneity limit prior to the static limit in the axial-vector vertex. Let us consider the fermion loop of an AVV diagram to which the axial-vector vertex is attached, whose contribution is denoted by $\Gamma$, with $n>1$ vector vertices as shown in Fig. 2. Note that the photon lines attached to this loop could be either internal lines or 
external ones. In the case $n=2$, Fig. 2 becomes the usual one-loop AVV diagram. In this loop, the axial-vector field with incoming momentum $k$ was inserted between vertices $\nu_{1}$ and $\nu_{2}$. By convention, the additional momentum $k$ running around the fermion loop from the axial-vector vertex would exit at vertex $\nu_{1}$. We have in CTP formalism

$$
\begin{aligned}
& \Gamma_{a_{1} c a_{2} \ldots a_{n}}^{\nu_{1} \rho \nu_{2} \cdots \nu_{n}}\left(k, k_{1}, \ldots, k_{n}\right) \\
& \quad=-(-i e)^{n} \int \frac{d p_{1}}{(2 \pi)^{4}} \operatorname{Tr}\left[\Gamma^{\rho 5} \eta_{c} S\left(p_{1}\right) \Gamma^{\nu_{1}} \eta_{a_{1}} S\left(p_{n}+k\right) \Gamma^{\nu_{n}} \eta_{a_{n}} S\left(p_{n-1}+k\right) \cdots S\left(p_{3}+k\right) \Gamma^{\nu_{3}} \eta_{a_{3}} S\left(p_{2}+k\right) \Gamma^{\nu_{2}} \eta_{a_{2}} S\left(p_{1}+k\right)\right],
\end{aligned}
$$

where $\Gamma^{\nu}, \Gamma^{\rho 5}$, and $S(p)$, given in (9) and (10), are the bare vector, axial-vector vertices, and quark propagator, respectively. Taking the divergence with respect to the axial-vector vertex and employing the identity

$$
S(p+k)\left(-i k_{\rho}\right) \Gamma^{\rho 5} \eta_{a} S(p)=\eta_{a} \gamma^{5} S(p)+S(p+k) \gamma^{5} \eta_{a}
$$

for massless fermions, we have

$$
\begin{aligned}
& i k_{\rho} \Gamma_{a_{1} c a_{2} \ldots a_{n}}^{\nu_{1} \rho \nu_{2} \cdots \nu_{n}}\left(k, k_{1}, \ldots, k_{n}\right) \\
& \quad=(-i e)^{n} \int \frac{d p_{1}}{(2 \pi)^{4}} \operatorname{Tr}\left[\Gamma^{\nu_{2}} \eta_{a_{2}} \eta_{c} \gamma^{5}\left(S\left(p_{1}\right)-S\left(p_{1}+k\right)\right) \Gamma^{\nu_{1}} \eta_{a_{1}} S\left(p_{n}+k\right) \Gamma^{\nu_{n}} \eta_{a_{n}} S\left(p_{n-1}+k\right) \cdots S\left(p_{3}+k\right) \Gamma^{\nu_{3}} \eta_{a_{3}} S\left(p_{2}+k\right)\right] .
\end{aligned}
$$

Likewise, if the axial-vector vertex was inserted between vertices $\nu_{2}$ and $\nu_{3}$, we will have

$$
\begin{aligned}
& i k_{\rho} \Gamma_{a_{1} a_{2} c a_{3} \ldots a_{n}}^{\nu_{1} \nu_{2} \nu_{3} \ldots \nu_{n}}\left(k, k_{1}, \ldots, k_{n}\right) \\
& \quad=(-i e)^{n} \int \frac{d p_{1}}{(2 \pi)^{4}} \operatorname{Tr}\left[\Gamma^{\nu_{2}} \eta_{a_{2}} \eta_{c} \gamma^{5} S\left(p_{1}\right) \Gamma^{\nu_{1}} \eta_{a_{1}} S\left(p_{n}+k\right) \Gamma^{\nu_{n}} \eta_{a_{n}} S\left(p_{n-1}+k\right) \cdots S\left(p_{3}+k\right) \Gamma^{\nu_{3}} \eta_{a_{3}}\left(S\left(p_{2}\right)-S\left(p_{2}+k\right)\right)\right] .
\end{aligned}
$$

Therefore the first term in (51) will be canceled by the second term in (52). Similar cancellations take place between terms from other pairs of diagrams with adjacent insertions of axial-vector vertices. Summing over all $n$ insertions, we end up with

$$
\begin{array}{rl}
\sum_{i=1}^{n} & i k_{\rho} \Gamma_{a_{1} a_{2} \cdots a_{i} c a_{i+1} \cdots a_{n}}^{\nu_{1} \nu_{2} \cdots \nu_{i} \rho \nu_{i+1} \cdots \nu_{n}}\left(k, k_{1}, \ldots, k_{n}\right) \\
= & -(-i e)^{n} \int \frac{d p_{1}}{(2 \pi)^{4}} \operatorname{Tr}^{n}\left[\Gamma^{\nu_{2}} \eta_{a_{2}} \eta_{c} \gamma^{5} S\left(p_{1}+k\right) \Gamma^{\nu_{1}} \eta_{a_{1}} S\left(p_{n}+k\right) \Gamma^{\nu_{n}} \eta_{a_{n}} S\left(p_{n-1}+k\right) \cdots S\left(p_{3}+k\right) \Gamma^{\nu_{3}} \eta_{a_{3}} S\left(p_{2}+k\right)\right. \\
& \left.-\Gamma^{\nu_{2}} \eta_{a_{2}} \eta_{c} \gamma^{5} S\left(p_{1}\right) \Gamma^{\nu_{1}} \eta_{a_{1}} S\left(p_{n}\right) \Gamma^{\nu_{n}} \eta_{a_{n}} S\left(p_{n-1}\right) \cdots S\left(p_{3}\right) \Gamma^{\nu_{3}} \eta_{a_{3}} S\left(p_{2}\right)\right],
\end{array}
$$

where the number $i$ in the summation is modulo $n$. Shifting the integration variable from $p_{1}$ to $p_{1}+k$ in the second term, if it is legitimate, the two remaining terms cancel. As shown by Adler and Bardeen [32], the only circumstance that invalidates this momentum shift was the diagram with $n=2$. While the legitimacy of the momentum shifting can be restored by a UV regulator, say a Pauli-Villars regulator, the regulator mass invalidates (50) and gives rise to the anomalous Ward identity

$$
-i\left(k_{1}+k_{2}\right)_{\rho} \Gamma_{c a_{1} a_{2}}^{\rho \nu_{1} \nu_{2}}\left(k_{1}, k_{2}\right)= \begin{cases}\frac{e^{2}}{4 \pi^{2}} \epsilon^{\nu_{1} \nu_{2} \alpha \beta} k_{1 \alpha} k_{2 \beta}, & c=a_{1}=a_{2}=1, \\ -\frac{e^{2}}{4 \pi^{2}} \epsilon^{\nu_{1} \nu_{2} \alpha \beta} k_{1 \alpha} k_{2 \beta}, & c=a_{1}=a_{2}=2, \\ 0, & \text { otherwise. }\end{cases}
$$




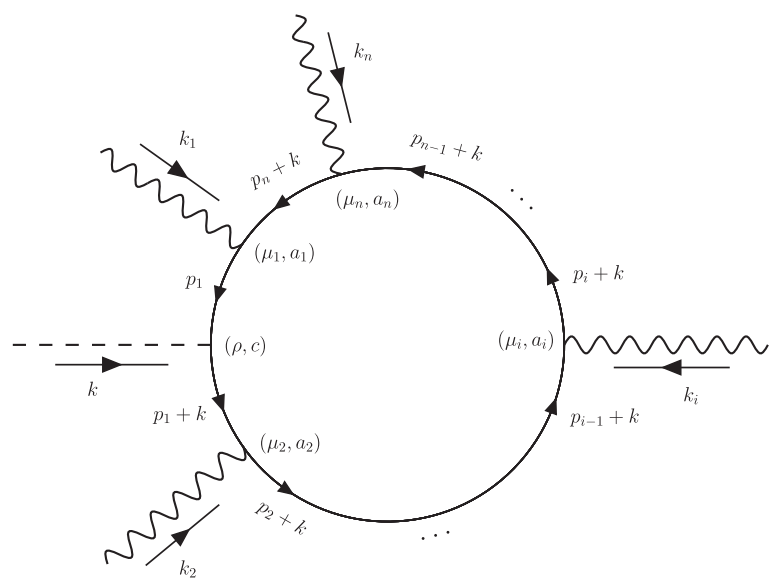

FIG. 2. The axial-vector vertex is attached to an internal fermion loop with $n>1$ vector vertices.

Consequently, the chiral magnetic current with this order of limits takes the form of (6) [33]. This results is in contrast to the null result in the order of limits, $\lim _{\mathbf{k} \rightarrow 0} \lim _{k^{0} \rightarrow 0}$, which is only valid in linear order of a small magnetic field momentum.

\section{A CONCRETE EXAMPLE}

In this section, we shall show a concrete example which demonstrates that dressing the fermion propagators could not smear the noncommutativity of the order of the spatial homogeneity limit and the static limit in the axial-vector vertex. One important lesson we learn is that in order to preserve the Ward identities for the vector or axial-vector vertex, $V_{\mu} \bar{\psi} \gamma^{\mu} \psi$ or $A_{\mu} \bar{\psi} \gamma^{\mu} \gamma^{5} \psi$, with dressed fermion propagators, the vertex should be dressed accordingly.

In CTP formulation, the Ward identities satisfied by the vector and axial-vector vertices are

$\mathcal{S}\left(p^{\prime}\right) \sigma_{1}\left(-i q_{\mu}\right) \Gamma^{\mu}\left(p^{\prime}, p\right) \sigma_{1} \mathcal{S}(p)=\mathcal{S}(p)-\mathcal{S}\left(p^{\prime}\right)$,

and

$\mathcal{S}\left(p^{\prime}\right) \sigma_{1}\left(-i q_{\mu}\right) \Gamma^{\mu 5}\left(p^{\prime}, p\right) \sigma_{1} \mathcal{S}(p)=\gamma_{5} \mathcal{S}(p)+\mathcal{S}\left(p^{\prime}\right) \gamma_{5}$, in the physical representation, where $\mathcal{S}(p)$ is the full fermion propagator carrying both spinor and CTP indices, $\Gamma^{\mu}, \Gamma^{\mu 5}$ are the amputated full vector and axial-vector vertex functions with $q=p^{\prime}-p$ the momentum flowing into it, and $\sigma_{1}$ is the Pauli matrix with respect to CTP indices, which always accompanies the CTP Green functions in physical representations. As shown, the Ward identity ties the longitudinal component of the vertex function to the propagator. Any dressing of the latter has to be reflected in the longitudinal component of the former. In case of the triangle diagrams underlying CME, while the longitudinal component of the photon vertex contributes to neither order of limit of the response to magnetic field, the longitudinal components of the axial-vector vertex does contribute to the order of limit in Sec. III B. The mechanism of removing the ambiguity of the orders of limit with respect to the magnetic field does not contradict to the vector Ward identity but fails here with respect to the axial-vector Ward identity.

In terms of the explicit form of the fermion propagator and axial-vector vertex function

$$
\mathcal{S}(p)=\left(\begin{array}{cc}
0 & \mathcal{S}_{A}(p) \\
\mathcal{S}_{R}(p) & \mathcal{S}_{C}(p)
\end{array}\right)
$$

and

$$
\Gamma^{\mu 5}\left(p^{\prime}, p\right)=\left(\begin{array}{cc}
0 & \Gamma_{A}^{\mu 5}\left(p^{\prime}, p\right) \\
\Gamma_{R}^{\mu 5}\left(p^{\prime}, p\right) & \Gamma_{C}^{\mu 5}\left(p^{\prime}, p\right)
\end{array}\right),
$$

the Ward identity (56) becomes

$$
\mathcal{S}_{A}\left(p^{\prime}\right)\left(-i q_{\mu}\right) \Gamma_{A}^{\mu 5}\left(p^{\prime}, p\right) \mathcal{S}_{A}(p)=\gamma^{5} \mathcal{S}_{A}(p)+\mathcal{S}_{A}\left(p^{\prime}\right) \gamma^{5},
$$

$$
\mathcal{S}_{R}\left(p^{\prime}\right)\left(-i q_{\mu}\right) \Gamma_{R}^{\mu 5}\left(p^{\prime}, p\right) \mathcal{S}_{R}(p)=\gamma^{5} \mathcal{S}_{R}(p)+\mathcal{S}_{R}\left(p^{\prime}\right) \gamma^{5},
$$

$$
\mathcal{S}_{R}\left(p^{\prime}\right)\left(-i q_{\mu}\right) \Gamma_{R A}^{\mu 5}\left(p^{\prime}, p\right) \mathcal{S}_{A}(p)=\gamma^{5} \mathcal{S}_{A}(p)+\mathcal{S}_{R}\left(p^{\prime}\right) \gamma^{5}
$$

where

$$
\Gamma_{R A}^{\mu 5}\left(p^{\prime}, p\right) \equiv \frac{\Gamma_{C}^{\mu 5}\left(p^{\prime}, p\right)+\left[1-2 f_{F}\left(p^{0 \prime}\right)\right] \Gamma_{A}^{\mu 5}\left(p^{\prime}, p\right)-\left[1-2 f_{F}\left(p^{0}\right)\right] \Gamma_{R}^{\mu 5}\left(p^{\prime}, p\right)}{2\left[f_{F}\left(p^{0}\right)-f_{F}\left(p^{0 \prime}\right)\right]},
$$

and the KMS relation

$$
\mathcal{S}_{C}(p)=\left[1-2 f_{F}\left(p^{0}\right)\right]\left[\mathcal{S}_{R}(p)-\mathcal{S}_{A}(p)\right]
$$

is employed. To the zeroth order, $\mathcal{S}_{A}(p)$ and $\mathcal{S}_{R}(p)$ are given by free propagators and $\Gamma_{A}^{\mu 5}\left(p^{\prime}, p\right)=\Gamma_{R}^{\mu 5}\left(p^{\prime}, p\right)=$ $\gamma^{\mu} \gamma^{5}$ and $\Gamma_{C}^{\mu 5}=0$. Consequently, $\Gamma_{R A}^{\mu 5}=\gamma^{\mu} \gamma^{5}$. Note that, in general, a full axial-vector vertex function $\Gamma^{\mu 5}\left(p^{\prime}, p\right)$ 
contains eight components with respect to the CTP indices. The external field, however, has equal values on both time branches in the CTP contour and thus the operator coupled to it only takes the physical component. Consequently, the number of components of the full axial-vector vertex reduces to four and it can be written by a $2 \times 2$ form as in (58). For the specific definitions of the components in (58), see the Appendix.

Let us consider the AVV three-point function shown in Fig. 3 with full propagators and a modified axial-vector vertex, and bare vector vertices. The retarded CME kernel implied by this diagram has the form

$$
\begin{aligned}
\mathcal{G}_{R}^{i j 0}(q, k)= & -i e^{2} \int \frac{d^{4} p}{(2 \pi)^{4}} \operatorname{Tr} \gamma^{i}\left[\mathcal{S}_{R}(p+q+k) \Gamma_{R}^{05}(p+q+k, p+q) \mathcal{S}_{R}(p+q) \gamma^{j} \mathcal{S}_{C}(p)\right. \\
& +\mathcal{S}_{R}(p+q+k) \Gamma_{C}^{05}(p+q+k, p+q) \mathcal{S}_{A}(p+q) \gamma^{j} \mathcal{S}_{A}(p) \\
& +\mathcal{S}_{C}(p+q+k) \Gamma_{A}^{05}(p+q+k, p+q) \mathcal{S}_{A}(p+q) \gamma^{j} \mathcal{S}_{A}(p) \\
& +\mathcal{S}_{R}(p+q+k) \Gamma_{R}^{05}(p+q+k, p+q) \mathcal{S}_{C}(p+q) \gamma^{j} \mathcal{S}_{A}(p) \\
& +\mathcal{S}_{C}(p+q+k) \gamma^{j} \mathcal{S}_{A}(p+k) \Gamma_{A}^{05}(p+k, p) \mathcal{S}_{A}(p)+\mathcal{S}_{R}(p+q+k) \gamma^{j} \mathcal{S}_{R}(p+k) \Gamma_{C}^{05}(p+k, p) \mathcal{S}_{A}(p) \\
& \left.+\mathcal{S}_{R}(p+q+k) \gamma^{j} \mathcal{S}_{C}(p+k) \Gamma_{A}^{05}(p+k, p) \mathcal{S}_{A}(p)+\mathcal{S}_{R}(p+q+k) \gamma^{j} \mathcal{S}_{R}(p+k) \Gamma_{R}^{05}(p+k, p) \mathcal{S}_{C}(p)\right] .
\end{aligned}
$$

Employing the KMS relation (61), we can rewrite the retarded CME kernel for a static magnetic field in terms of retarded and advanced propagators

$$
\begin{aligned}
\mathcal{G}_{R}^{i j 0}(q, k)= & -i e^{2} \int \frac{d^{4} p}{(2 \pi)^{4}} \operatorname{Tr} \gamma^{i}\left\{f _ { F } ( p ^ { 0 } ) \left[\mathcal{S}_{R}(p+q+k) \Gamma_{R}^{05}(p+q+k, p+q) \mathcal{S}_{R}(p+q) \gamma^{j} \mathcal{S}_{R}(p)\right.\right. \\
& \left.+\mathcal{S}_{R}(p+q+k) \gamma^{j} \mathcal{S}_{R}(p+k) \Gamma_{R}^{05}(p+k, p) \mathcal{S}_{R}(p)\right] \\
& +\left[f_{F}\left(p^{0}+q^{0}\right)-f_{F}\left(p^{0}\right)\right]\left[\mathcal{S}_{R}(p+q+k) \Gamma_{R A}^{05}(p+q+k, p+q) \mathcal{S}_{A}(p+q) \gamma^{j} \mathcal{S}_{A}(p)\right. \\
& \left.-\mathcal{S}_{R}(p+q+k) \gamma^{j} \mathcal{S}_{R}(p+k) \Gamma_{R A}^{05}(p+k, p) \mathcal{S}_{A}(p)\right] \\
& -f_{F}\left(p^{0}+k^{0}\right)\left[\mathcal{S}_{A}(p+q+k) \Gamma_{A}^{05}(p+q+k, p+q) \mathcal{S}_{A}(p+q) \gamma^{j} \mathcal{S}_{A}(p)\right. \\
& \left.\left.+\mathcal{S}_{A}(p+q+k) \gamma^{j} \mathcal{S}_{A}(p+q) \Gamma_{A}^{05}(p+q, p) \mathcal{S}_{A}(p)\right]\right\},
\end{aligned}
$$

with $q=(0, \mathbf{q})$ and $k=\left(k^{0}, \mathbf{k}\right)$, in parallel to the one-loop expression (17). Considering a homogeneous axial chemical potential, i.e., $k=\left(k^{0}, \mathbf{0}\right)$, and taking the divergence at the axial-vector vertex (projecting out the longitudinal component), we end up with

$$
\begin{aligned}
k^{0} \mathcal{G}_{R}^{i j 0}= & e^{2} \int \frac{d^{4} p}{(2 \pi)^{4}}\left\{f_{F}\left(p^{0}\right) \operatorname{Tr} \gamma^{i} \gamma^{5}\left[\mathcal{S}_{R}(p+q) \gamma^{j} \mathcal{S}_{R}(p)-S_{A}(p+q) \gamma^{j} \mathcal{S}_{A}(p)\right]\right. \\
& \left.-f_{F}\left(p^{0}+k^{0}\right) \operatorname{Tr} \gamma^{i} \gamma^{5}\left[\mathcal{S}_{R}(p+q+k) \gamma^{j} \mathcal{S}_{R}(p+k)-\mathcal{S}_{A}(p+q+k) \gamma^{j} \mathcal{S}_{A}(p+k)\right]\right\}
\end{aligned}
$$

where the Ward identities (59) were employed. It follows that

$$
\lim _{k^{0} \rightarrow 0} \lim _{\mathbf{k} \rightarrow 0} \mathcal{G}_{R}^{i j 0}(q, k)=e^{2} \int \frac{d^{4} p}{(2 \pi)^{4}} \frac{\partial}{\partial p^{0}}\left\{f_{F}\left(p^{0}\right) \operatorname{Tr} \gamma^{i}\left[\mathcal{S}_{R}(p+q) \gamma^{5} \gamma^{j} \mathcal{S}_{R}(p)-\mathcal{S}_{A}(p+q) \gamma^{5} \gamma^{j} \mathcal{S}_{A}(p)\right]\right\},
$$

in parallel to (27). In a proper regularization, for instance the PV regularization, the momentum shift will be legitimate and the two terms cancel out, or, equivalently, (65) vanish. The regulator term will contribute to the nonzero current (6).

To be specific, let us consider the dressed propagator (29). It follows from (59) and (60) for $p^{\prime}-p=\left(k^{0}, \mathbf{0}\right)$ that

$$
\begin{aligned}
& \Gamma_{R}^{05}\left(p^{\prime}, p\right)=\Gamma_{A}^{05}\left(p^{\prime}, p\right)=\gamma^{0} \gamma^{5}, \\
& \Gamma_{R A}^{05}\left(p^{\prime}, p\right)=\gamma^{0} \gamma^{5}+\frac{2 i}{k^{0} \tau} \gamma^{0} \gamma^{5}, \\
& \Gamma_{C}^{05}\left(p^{\prime}, p\right)=\frac{4 i}{k^{0} \tau}\left[f_{F}\left(p^{0}\right)-f_{F}\left(p^{0}+k^{0}\right)\right] \gamma^{0} \gamma^{5} .
\end{aligned}
$$




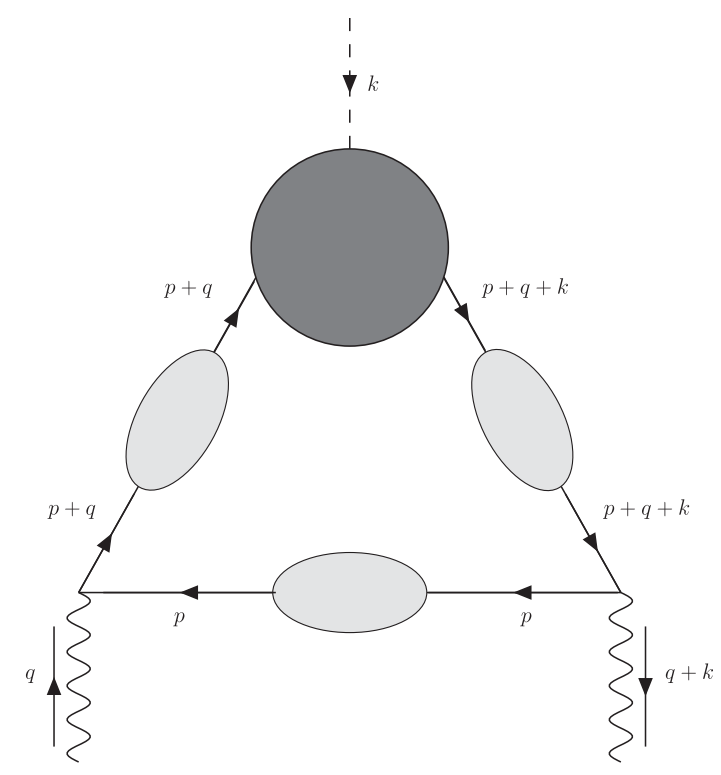

FIG. 3. Subdiagrams with a modified axial-vector vertex and full fermion propagators, but bare vector vertices. There is a second set of subdiagrams with the photon four-momenta and polarization indices interchanged.

Consequently, the role of the pinching singularity with the bare propagator and axial-vector vertex is taken over by the $1 / k^{0}$ singularity of the dressed axial-vector vertex function $\Gamma_{R A}^{05}\left(p^{\prime}, p\right)$ in the limit of $k^{0} \rightarrow 0$. In another word, it is precisely this singularity that facilitates the reduction from (63) to (65), in the limit $k^{0} \rightarrow 0$, in parallel with the reduction from (17) to (27) in the one-loop case.

We have to emphasize that Fig. 3 only represents a subset of diagrams underlying the radiative corrections to the AVV triangle whose integrand adds up to a total derivative with respect to the energy running through the Fermion loop and thereby contributing to the nonrenormalization of the

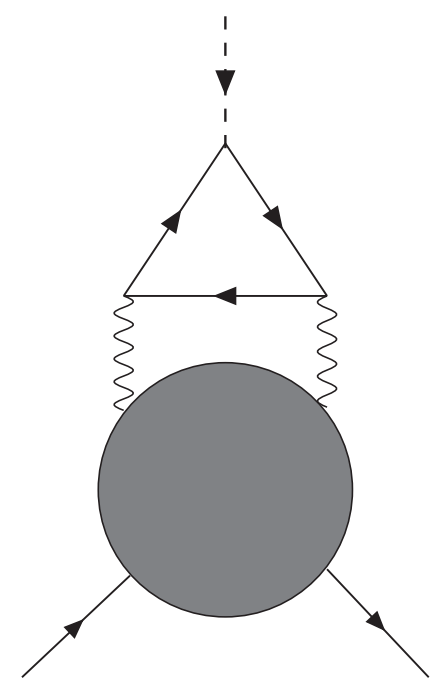

FIG. 4. An axial-vector vertex not considered in Fig. 3. anomaly independent of other diagrams. The dressed axial-vector vertex in Fig. 3 excludes the diagrams in Fig. 4, which adds another term to the RHS of (56) because of the anomaly [31,32]. The photon vertices in Fig. 3 remain undressed. Dressing the photon vertices amounts to introducing more bare photon vertices with internal photon lines attached to them and the logic from (57) to (64) is expected to be carried through. On the other hand, even with dressed photon vertices, the diagrams included in Fig. 3 do not cover all radiative corrections to AVV triangle. An example not included in Fig. 3 can be found in Refs. [34,35]. Nevertheless, the selection of the subset of diagrams in Fig. 3 demonstrates that modifying the fermion propagator with damping term does not remove ambiguity of the infrared limit of the four-momentum pertaining to the axial chemical potential.

\section{CONCLUSIONS AND OUTLOOKS}

In this paper, we studied the noncommutativity of different orders of zero energy-momentum limit pertaining to the axial chemical potential in the chiral magnetic effect. The vanishing CME in the limit that the static limit was taken prior to the homogeneous limit was proved in general by an argument similar to the Coleman-Hill theorem for a static external magnetic field. For the opposite limit that the homogeneous limit is taken first the nonvanishing CME was a consequence of the nonrenormalization of chiral anomaly. While the nonrenormalization of chiral anomaly is valid for arbitrary external momenta, the Coleman-Hill theorem applies only in the limit $\mathbf{q} \rightarrow 0$ with $\mathbf{q}$ the spatial momentum of the external magnetic field. A possible caveat of the Coleman-Hill theorem is the infrared divergence at a nonzero temperature when more and more internal gluon lines are introduced. But the lattice simulation indicates a nonzero magnetic mass of gluons [36-38], which serves an infrared cutoff. In addition, a recent calculation based on holography shows that such a noncommutativity stays in strongly coupled $N=4$ super Yang-Mills theory [39].

At one-loop level, the noncommutativity of different orders of zero momentum limit is originated from the pinching of the poles of the retarded and advanced fermion propagators convoluting with the difference of the distribution functions of this fermion pair. It is tentative to smear the pinching singularity by introducing a finite damping term to the fermion propagators and thereby removing the noncommutativity between different orders of limits. The mechanism works for the photon vertex attached to the magnetic field and the infrared ambiguity is indeed removed. As we have shown in this work, this approach does not work for the axial-vector vertex. The physical reason is that modifying the propagator alone would violate the vector and axial-vector Ward identities, which requires a corresponding modification of the longitudinal component of the vector and axial-vector vertices with respect to 
the four-momentum transfer. For the vector vertex attached to the magnetic field, according to (3), only the transverse component matters in either order of limits and the issue does not arise. For the axial-vector vertex, the static limit after homogeneity limit picks up the longitudinal component and the modified vertex contributes. We demonstrate this point by a subset of diagrams contributing to CME and show explicitly that the difference between the two orders of limits remains as expected.

Our work at this stage is of theoretical value only. In view of the dynamical nature of the chirality imbalance in realistic heavy ion collisions, it is important to explore under which order of limits the constant $\mu_{5}$ approximation better describes the phenomenology of the chiral magnetic effect there.

\section{ACKNOWLEDGMENTS}

The works of D.-f. H. and H.-c. R. are in part supported by the NSFC Grants No. 11735007, No. 11890710 and No. 11890711. B. F.'s work was supported in part by the NSFC Grants No. 12075093 and No. 11535005.

\section{APPENDIX: GREEN FUNCTIONS AND VERTICES IN CLOSED-TIME PATH FORMALISM}

As the closed-time path (CTP) Green's function is less well known than the Green's functions underlying the Feynman diagrams and Matsubara diagrams, we provide some background behind the CTP formalism employed in this work. A systematic discussion of CTP formulation and its applications can be found in [25].

The CTP Green's functions are generated by a path integral whose action is the integration of the classical Lagrangian along a closed time path which consists of a forward branch, $\int_{-\infty}^{\infty} d t(\ldots)$ and a backward branch, $\int_{\infty}^{-\infty} d t(\ldots)$. The number of degrees of freedom is thereby doubled. The quantum field operator in CTP is denoted by $\phi_{\alpha}(x)$ with $\alpha=1,2$ labeling the forward and backward branches. The time-ordering operator underlying the CTP Green's functions becomes the path-ordering along the closed time path, i.e., ordinary time ordering along the forward branch and antitime ordering along the backward branch with the backward branch preceding the forward branch. The two-point Green function of operators $A_{\alpha}\left(t_{1}\right)$ and $B_{\beta}\left(t_{2}\right)$ is defined as

$$
D_{\alpha \beta}\left(t_{1}, t_{2}\right)=\left\langle T_{p}\left(A_{\alpha}\left(t_{1}\right) B_{\beta}\left(t_{2}\right)\right)\right\rangle,
$$

where $T_{p}$ enforces the path-ordering operator along the CTP contour and the dependence on the spatial and internal coordinates is suppressed for clarity.

It is convenient to write the two-point function in a $2 \times 2$ matrix form, named as the single-time representation in [25], i.e.,

$$
D=\left(\begin{array}{c}
D_{11}, D_{12} \\
D_{21}, D_{22}
\end{array}\right)
$$

with

$$
\begin{gathered}
D_{11}\left(t_{1}, t_{2}\right)=\left\langle T\left(A\left(t_{1}\right) B\left(t_{2}\right)\right)\right\rangle, \\
D_{12}\left(t_{1}, t_{2}\right)=\left\langle B\left(t_{2}\right) A\left(t_{1}\right)\right\rangle, \\
D_{21}\left(t_{1}, t_{2}\right)= \pm\left\langle A\left(t_{1}\right) B\left(t_{2}\right)\right\rangle, \\
D_{22}\left(t_{1}, t_{2}\right)=\left\langle\tilde{T}\left(A\left(t_{1}\right) B\left(t_{2}\right)\right)\right\rangle,
\end{gathered}
$$

where $T$ is the usual time-ordering operator, while $\tilde{T}$ is the antitime-ordering operator. Whenever " \pm " or "干" shows up, the upper sign refers to bosons and the lower sign to fermions. The four components in the matrix form (A2) are not independent and satisfy the following identity:

$$
D_{11}+D_{22}=D_{12}+D_{21}
$$

As shown in (A3), once the operators are placed explicitly in the order with backward branch preceding the forward branch, the branch indices are removed since both $\phi_{1}(t)$ and $\phi_{2}(t)$ corresponds to the same Hilbert space operator. In particular, we have $T_{p}\left(\phi_{1}(t)\right)=T_{p}\left(\phi_{2}(t)\right)=\phi(t)$.

The CTP Green functions are also defined with respect to "physical" field operators

$$
\begin{aligned}
\phi_{\Delta}(t) & =\phi_{1}(t)-\phi_{2}(t), \\
\phi_{c}(t) & =\frac{1}{2}\left(\phi_{1}(t)+\phi_{2}(t)\right) .
\end{aligned}
$$

Consequently, the physical representation (with $\Delta$ and $c$ indices) of the CTP Green function can be obtained from (A2) by an orthogonal transformation, i.e.,

$$
D=V^{-1} \mathcal{D} V,
$$

with

$V=\frac{1}{\sqrt{2}}\left(1-i \sigma^{2}\right)=\frac{1}{\sqrt{2}}\left(\begin{array}{rr}1 & -1 \\ 1 & 1\end{array}\right), \quad V^{-1}=\frac{1}{\sqrt{2}}\left(\begin{array}{rr}1 & 1 \\ -1 & 1\end{array}\right)$,

and we end up with

$$
\mathcal{D}=\left(\begin{array}{cc}
0 & D_{A} \\
D_{R} & D_{C}
\end{array}\right)
$$

where

$$
D_{R}\left(t_{1}, t_{2}\right)=\theta(12)\left\langle\left[A\left(t_{1}\right), B\left(t_{2}\right)\right]_{\mp}\right\rangle,
$$




$$
\begin{gathered}
D_{A}\left(t_{1}, t_{2}\right)=-\theta(21)\left\langle\left[A\left(t_{1}\right), B\left(t_{2}\right)\right]_{\mp}\right\rangle, \\
D_{C}\left(t_{1}, t_{2}\right)=\left\langle\left[A\left(t_{1}\right), B\left(t_{2}\right)\right]_{ \pm}\right\rangle,
\end{gathered}
$$

where $\theta(12)$ is the step function, which equals one if $t_{1}>t_{2}$ and vanishes otherwise, and $[\cdots]_{\mp}$ stands for commutator and anticommutator. $D_{R}, D_{A}$, and $D_{C}$ are the retarded, advanced and correlation functions, respectively, and they satisfy the KMS relation

$$
D_{C}(p)=\left[1-2 f\left(p^{0}\right)\right]\left[D_{R}(p)-D_{A}(p)\right]
$$

with $f\left(p^{0}\right)=1 /\left(e^{\beta p^{0}} \mp 1\right)$ the Bose-Einstein or FermiDirac distribution functions for the bosonic or fermionic fields, respectively. The same orthogonal transformation (A6) also converts the signature matrix of time integration along the CTP contour, $\sigma_{3}$ in the single time representation to $\sigma_{1}$ in the physical representation.

It is instructive to verify the structure of (A8) directly from definition since the methodology can be readily extended to the three-point function considered in this work. The $\Delta \Delta$-component of (A8) takes the form

$$
\begin{aligned}
\left\langle T_{p}\left(A_{\Delta}\left(t_{1}\right) B_{\Delta}\left(t_{2}\right)\right)\right\rangle & =\theta(12)\left\langle T_{p}\left(A_{\Delta}\left(t_{1}\right) B_{\Delta}\left(t_{2}\right)\right)\right\rangle \pm \theta(21)\left\langle T_{p}\left(B_{\Delta}\left(t_{2}\right) A_{\Delta}\left(t_{1}\right)\right)\right\rangle \\
& =\theta(12)\left\langle T_{p}\left(A_{\Delta}\left(t_{1}\right)\left(B_{1}\left(t_{2}\right)-B_{2}\left(t_{2}\right)\right)\right)\right\rangle \pm \theta(21)\left\langle T_{p}\left(B_{\Delta}\left(t_{2}\right)\left(A_{1}\left(t_{1}\right)-A_{2}\left(t_{1}\right)\right)\right)\right\rangle .
\end{aligned}
$$

Looking at the first term on RHS, $t_{2}$ is the earliest moment, therefore, $B_{1}\left(t_{2}\right)$ should reside at the rightmost position and $B_{2}\left(t_{2}\right)$ at the leftmost position, i.e.,

$$
1 \text { st term }=\theta(12)\left\langle\left(A_{1}\left(t_{1}\right)-A_{2}\left(t_{1}\right)\right) B_{1}\left(t_{2}\right) \mp B_{2}\left(t_{2}\right)\left(A_{1}\left(t_{1}\right)-A_{2}\left(t_{2}\right)\right)\right\rangle=\theta(12)\left\langle\left[A\left(t_{1}\right)-A\left(t_{1}\right), B\left(t_{2}\right)\right]_{\mp}\right\rangle=0 .
$$

The same logic renders the second term vanish as well and we find $\left\langle T_{p}\left(A_{\Delta}\left(t_{1}\right) B_{\Delta}\left(t_{2}\right)\right)\right\rangle=0$. Next, let us consider the $\Delta c$-component. We have

$$
\left\langle T_{p}\left(A_{\Delta}\left(t_{1}\right) B_{c}\left(t_{2}\right)\right)\right\rangle=\frac{1}{2} \theta(12)\left\langle T_{p}\left[\left(A_{1}\left(t_{1}\right)-A_{2}\left(t_{1}\right)\right)\left(B_{1}\left(t_{2}\right)+B_{2}\left(t_{2}\right)\right)\right]\right\rangle \pm \frac{1}{2} \theta(21)\left\langle T_{p}\left[\left(B_{1}\left(t_{2}\right)+B_{2}\left(t_{2}\right)\right)\left(A_{1}\left(t_{1}\right)-A_{2}\left(t_{1}\right)\right)\right]\right\rangle,
$$

where

$$
1 \text { st term }=\frac{1}{2} \theta(12)\left\langle\left(A_{1}\left(t_{1}\right)-A_{2}\left(t_{1}\right)\right) B_{1}\left(t_{2}\right) \pm B_{2}\left(t_{2}\right)\left(A_{1}\left(t_{1}\right)-A_{2}\left(t_{2}\right)\right)\right\rangle=\frac{1}{2} \theta(12)\left\langle\left[\left(A\left(t_{1}\right)-A\left(t_{1}\right)\right), B\left(t_{2}\right)\right]_{\mp}\right\rangle=0,
$$

and

$$
\text { 2nd term }=\frac{1}{2} \theta(21)\left\langle\left(B_{1}\left(t_{2}\right)+B_{2}\left(t_{2}\right)\right) A_{1}\left(t_{1}\right) \mp A_{2}\left(t_{1}\right)\left(B_{1}\left(t_{2}\right)+B_{2}\left(t_{2}\right)\right)\right\rangle=\theta(21)\left\langle\left[B\left(t_{2}\right), A\left(t_{1}\right)\right]_{\mp}\right\rangle,
$$

which gives rise to (A9b). The same manipulation, when applied to the $c \Delta$-component, leads to (A9a).

This type of reduction extends readily to $n$-point Green function with the recipe: 1) Write down all possible orders of the time variables by inserting the identity

$$
1=\sum_{\mathcal{P}} \theta\left(p_{1} p_{2} \ldots p_{n-1} p_{n}\right)
$$

with $\theta(12 \ldots n)=\theta(12) \theta(23) \ldots \theta((n-1) n)$ and the sum extends to all permutation of $1,2, \ldots, n$. 2) Time order the operators inside $T_{p}(\ldots)$, and 3$)$ Remove the operators one by one from $T_{p}(\ldots)$ according to their location in the forward or backward time branches. As an illustration, we consider the following three-point function:

$$
\left\langle T_{p}\left(A_{\Delta}\left(t_{1}\right) B_{\alpha}\left(t_{2}\right) C_{\beta}\left(t_{3}\right)\right)\right\rangle,
$$

with $\alpha, \beta=\Delta$ or $c$. The RHS of (A16) consists of six permutations of the time ordering. To have a nonzero $T_{p}$ product, the latest time must be associated with the $c$-component. Consequently 


$$
\left\langle T_{p}\left(A_{\Delta}\left(t_{1}\right) B_{\Delta}\left(t_{2}\right) C_{\Delta}\left(t_{3}\right)\right)\right\rangle=0 .
$$

For the $T_{p}$ product with one operator in $c$-component, we have

$$
\begin{aligned}
\left\langle T_{p}\left(A_{\Delta}\left(t_{1}\right) B_{\Delta}\left(t_{2}\right) C_{c}\left(t_{3}\right)\right)\right\rangle & =\theta(312)\left\langle T_{p}\left(C_{c}\left(t_{3}\right) A_{\Delta}\left(t_{1}\right) B_{\Delta}\left(t_{2}\right)\right)\right\rangle \pm \theta(321)\left\langle T_{p}\left(C_{c}\left(t_{3}\right) B_{\Delta}\left(t_{2}\right) A_{\Delta}\left(t_{1}\right)\right)\right\rangle \\
& =\theta(312)\left\langle\left[\left[C\left(t_{3}\right), A\left(t_{1}\right)\right]_{\mp}, B\left(t_{2}\right)\right]_{-}\right\rangle \pm \theta(321)\left\langle\left[\left[C\left(t_{3}\right), B\left(t_{2}\right)\right]_{\mp}, A\left(t_{1}\right)\right]_{-}\right\rangle,
\end{aligned}
$$

and

$$
\begin{aligned}
\left\langle T_{p}\left(A_{\Delta}\left(t_{1}\right) B_{c}\left(t_{2}\right) C_{\Delta}\left(t_{3}\right)\right)\right\rangle & = \pm \theta(213)\left\langle T_{p}\left(B_{c}\left(t_{2}\right) A_{\Delta}\left(t_{1}\right) C_{\Delta}\left(t_{3}\right)\right)\right\rangle+\theta(231)\left\langle T_{p}\left(B_{c}\left(t_{2}\right) C_{\Delta}\left(t_{3}\right) A_{\Delta}\left(t_{1}\right)\right)\right\rangle \\
& = \pm \theta(213)\left\langle\left[\left[B\left(t_{2}\right), A\left(t_{1}\right)\right]_{\mp}, C\left(t_{3}\right)\right]_{-}\right\rangle+\theta(231)\left\langle\left[\left[B\left(t_{2}\right), C\left(t_{3}\right)\right]_{\mp}, A\left(t_{1}\right)\right]_{-}\right\rangle,
\end{aligned}
$$

where, without loss of generality, we assume that the operators $A(t), B(t)$ and $C(t)$ are simultaneously bosonic or fermionic ones. It is straightforward to figure out the relative signs in (A19) and (A20) if one or more operators are in different type.

Now we are equipped to analyze the structure of the threepoint functions encountered in this work. While a three-point function has eight CTP components in general, not all components contribute to our case. If an operator underlying the three point-function couples to an external field, only $\Delta$ component of the operator contributes since the external field takes equal values on both time branches. For the AVV function, we associate $A\left(t_{1}\right)$ with the axial-vector current density coupling with the axial chemical potential, $B\left(t_{2}\right)$ with the electric current coupling with gauge potential underlying the magnetic field, and $C\left(t_{3}\right)$ with the electric current to be measured. Thereby only two CTP components are left over, i.e., $\Delta \Delta \Delta$ - and $\Delta \Delta c$-components with the former one vanishing in according to (A18). Consequently

$\left\langle T_{p}\left(A_{\Delta}\left(t_{1}\right) B_{\Delta}\left(t_{2}\right) C_{c}\left(t_{3}\right)\right)\right\rangle=\left\langle T_{p}\left(A_{\Delta}\left(t_{1}\right) B_{\Delta}\left(t_{2}\right) C_{1}\left(t_{3}\right)\right)\right.$, which, upon applying the Wick theorem, gives rise to the retarded kernel (12) or (62).

Coming to the dressed axial-vector vertex in (58), $A_{\mu} \bar{\psi} \gamma^{\mu} \psi$, we associate $A\left(t_{1}\right)$ with the axial vector current, and $B\left(t_{2}\right)$ and $C\left(t_{3}\right)$ fermionic fields. Only $A\left(t_{1}\right)$ couples to the external $\mu_{5}$, we are left with four CTP components, which can be packed in a $2 \times 2$ matrix

$$
\left(\begin{array}{cc}
\left\langle T_{p}\left(A_{\Delta}\left(t_{1}\right) B_{\Delta}\left(t_{2}\right) C_{\Delta}\left(t_{3}\right)\right)\right\rangle & \left\langle T_{p}\left(A_{\Delta}\left(t_{1}\right) B_{\Delta}\left(t_{2}\right) C_{c}\left(t_{3}\right)\right)\right\rangle \\
\left\langle T_{p}\left(A_{\Delta}\left(t_{1}\right) B_{c}\left(t_{2}\right) C_{\Delta}\left(t_{3}\right)\right)\right\rangle & \left\langle T_{p}\left(A_{\Delta}\left(t_{1}\right) B_{c}\left(t_{2}\right) C_{c}\left(t_{3}\right)\right)\right\rangle
\end{array}\right),
$$

where the upper left element vanishes, resonating the structure of (58). Notice that the vertex function is obtained by amputating the two fermion legs and the amputation leaves the structure intact.

A comprehensive discussion of the general multipoint Green functions and vertices in the physical representation can be found in [25].
[1] D. E. Kharzeev, L. D. McLerran, and H. J. Warringa, The effects of topological charge change in heavy ion collisions: 'Event by event $\mathrm{P}$ and $C P$ violation', Nucl. Phys. A803, 227 (2008).

[2] K. Fukushima, D. E. Kharzeev, and H. J. Warringa, The chiral magnetic effect, Phys. Rev. D 78, 074033 (2008).

[3] D. E. Kharzeev and H. J. Warringa, Chiral magnetic conductivity, Phys. Rev. D 80, 034028 (2009).

[4] STAR Collaboration, Azimuthal Charged-Particle Correlations and Possible Local Strong Parity Violation, Phys. Rev.
Lett. 103, 251601 (2009); Observation of charge-dependent azimuthal correlations and possible local strong parity violation in heavy ion collisions, Phys. Rev. C 81, 054908 (2010); Fluctuations of charge separation perpendicular to the event plane and local parity violation in $\sqrt{s_{N N}}=200 \mathrm{GeV}$ $\mathrm{Au}+\mathrm{Au}$ collisions at the BNL relativistic heavy ion collider, Phys. Rev. C 88, 064911 (2013); Measurement of charge multiplicity asymmetry correlations in high-energy nucleusnucleus collisions at $\sqrt{s_{N N}}=200 \mathrm{GeV}$, Phys. Rev. C 89, 044908 (2014); Beam-Energy Dependence of Charge 
Separation Along the Magnetic Field in $\mathrm{Au}+\mathrm{Au}$ Collisions at RHIC, Phys. Rev. Lett. 113, 052302 (2014).

[5] ALICE Collaboration, Charge Separation Relative to the Reaction Plane in Pb-Pb Collisions at $\sqrt{s_{N N}}=2.76 \mathrm{TeV}$, Phys. Rev. Lett. 110, 012301 (2013); Constraining the magnitude of the chiral magnetic effect with event shape engineering in $\mathrm{Pb}-\mathrm{Pb}$ collisions at $\sqrt{s_{N N}}=2.76 \mathrm{TeV}$, Phys. Lett. B 777, 151 (2018).

[6] CMS Collaboration, Observation of Charge-Dependent Azimuthal Correlations in $\mathrm{p}-\mathrm{Pb}$ Collisions and Its Implication for the Search for the Chiral Magnetic Effect, Phys. Rev. Lett. 118, 122301 (2017); Constraints on the chiral magnetic effect using charge-dependent azimuthal correlations in $\mathrm{pPb}$ and $\mathrm{PbPb}$ collisions at the $\mathrm{CERN}$ large hadron collider, Phys. Rev. C 97, 044912 (2018).

[7] D. T. Son and B. Z. Spivak, Chiral anomaly and classical negative magnetoresistance of Weyl metals, Phys. Rev. B 88, 104412 (2013).

[8] Q. Li, D. E. Kharzeev, C. Zhang, Y. Huang, I. Pletikosic, A. V. Fedorov, R. D. Zhong, J. A. Schneeloch, G. D. Gu, and T. Valla, Chiral magnetic effect in ZrTe5, Nat. Phys. 12, 550 (2016).

[9] X.-c. Huang et al., Observation of the Chiral-AnomalyInduced Negative Magnetoresistance in 3D Weyl Semimetal TaAs, Phys. Rev. X 5, 031023 (2015).

[10] K. Taguchi and Y. Tanaka, Axial current driven by magnetization dynamics in Dirac semimetals, Phys. Rev. B 91, 054422 (2015).

[11] D. E. Kharzeev, J. Liao, S. A. Voloshin, and G. Wang, Chiral magnetic and vortical effects in high-energy nuclear collisions-A status report, Prog. Part. Nucl. Phys. 88, 1 (2016).

[12] F.-Q. Wang and J. Zhao, Search for the chiral magnetic effect in heavy ion collisions, Nuclear Science and Techniques 29, 179 (2018).

[13] V. Koch, S. Schlichting, V. Skokov, P. Sorensen, J. Thomas, S. Voloshin, G. Wang, and H.-U. Yee, Chiral magnetic effect task force report, Chin. Phys. C 41, 072001 (2017).

[14] Y.-c Liu and X.-g Huang, Anomalous chiral transports and spin polarization in heavy-ion collisions, Nuclear Science and Techniques 31, 56 (2020).

[15] D-f Hou, H. Liu, and H-c Ren, Some field theoretic issues regarding the chiral magnetic effect, J. High Energy Phys. 05 (2011) 046.

[16] B. Feng, D-f. Hou, H. Liu, H-c. Ren, P-p. Wu, and Y. Wu, Chiral magnetic effect in a lattice model, Phys. Rev. D 95, 114023 (2017).

[17] D. Satow and H.-U. Yee, Chiral magnetic effect at weak coupling with relaxation dynamics, Phys. Rev. D 90, 014027 (2014).

[18] H.-U. Yee, Holographic chiral magnetic conductivity, J. High Energy Phys. 11 (2009) 085.

[19] D. T. Son and N. Yamamoto, Berry Curvature, Triangle Anomalies, and the Chiral Magnetic Effect in Fermi Liquids, Phys. Rev. Lett. 109, 181602 (2012).

[20] M. A. Stephanov and Y. Yin, Chiral Kinetic Theory, Phys. Rev. Lett. 109, 162001 (2012).

[21] J-w. Chen, S. Pu, Q. Wang, and X-n. Wang, Berry Curvature and Four-Dimensional Monopoles in the Relativistic Chiral Kinetic Equation, Phys. Rev. Lett. 110, 262301 (2013).
[22] D. T. Son and N. Yamamoto, Kinetic theory with Berry curvature from quantum field theories, Phys. Rev. D 87, 085016 (2013).

[23] D. E. Kharzeev, M. A. Stephanov, and H.-U. Yee, Anatomy of the chiral magnetic effect in and out of equilibrium, Phys. Rev. D 95, 051901(R) (2017).

[24] S. Golkar and D. T. Son, (Non)-renormalization of the chiral vortical effect coefficient, J. High Energy Phys. 02 (2015) 169.

[25] K.-c. Chou, Z.-b. Su, B.-1 Hao, and L. Yu, Equilibrium and nonequilibrium formalisms made unified, Phys. Rep. 118, 1 (1985).

[26] This relation can be deduced from the Ward identities (59) with free propagators and vertex or can be verified explicitly.

[27] M. A. Valle Basagoiti, Transport coefficients and ladder summation in hot gauge theories, Phys. Rev. D 66, 045005 (2002).

[28] J.-S Gagnon and S. Jeon, Leading-order calculation of electric conductivity in hot quantum electrodynamics from diagrammatic methods, Phys. Rev. D 75, 025014 (2007); Erratum, Phys. Rev. D 76, 089902 (2007).

[29] See, e.g., Sec. II of L. Alvarez-Gaume and E. Witten, Nucl. Phys. B234, 269 (1983) for general regularization schemes of axial anomalies.

[30] S. Coleman and B. Hill, No more corrections to the topological mass term in QED3, Phys. Lett. 159B, 184 (1985).

[31] S. L. Adler, Axial-vector vertex in spinor electrodynamics, Phys. Rev. 177, 2426 (1969).

[32] S. L. Adler and W. A. Bardeen, Absence of higher-order corrections in the anomalous axial-vector divergence equation, Phys. Rev. 182, 1517 (1969).

[33] Incidentally, a radiative correction to the anomalous Ward identity stemming from a three-loop diagram with the two photons coming from the triangle diagram rescattered was found in Ref. [34]. The same diagram contributes to the CME current at zero temperature but the contribution vanishes in the limit considered here at a nonzero temperature for a static magnetic field. See Ref. [35] for details.

[34] A. A. Ansel'm and A. A. Iogansen, Radiative corrections to the axial anomaly, Zh. Eksp. Teor. Fiz. 96, 1181 (1989) [Sov. Phys. JETP 96, 670 (1989)].

[35] B. Feng, D.-f Hou, and H.-c Ren, QED radiative corrections to chiral magnetic effect, Phys. Rev. D 99, 036010 (2019).

[36] Y. Maezawa, S. Aoki, S. Ejiri, T. Hatsuda, N. Ishii, K. Kanaya, N. Ukita, and T. Umeda, Electric and magnetic screening masses at finite temperature from generalized Polyakov-line correlations in two-flavor lattice QCD, Phys. Rev. D 81, 091501 (2010).

[37] S. Borsanyi, Z. Fodor, S. D. Katz, A. Pásztor, K. K. Szabó, and C. Török, Static $\bar{Q} Q$ pair free energy and screening masses from correlators of Polyakov loops: Continuum extrapolated lattice results at the QCD physical point, J. High Energy Phys. 04 (2015) 138.

[38] C. Bonati, M. D’Elia, M. Mariti, M. Mesiti, F. Negro, A. Rucci, and F. Sanfilippo, Screening masses in strong external magnetic fields, Phys. Rev. D 95, 074515 (2017).

[39] L. Yin, D.-f. Hou, and H-c Ren, arXiv:2102.04851. 